\title{
Multimodal Characterization of Graphene
}

\author{
Stefanie Freitag ${ }^{1}$ \\ ${ }^{1}$ Carl Zeiss Microscopy GmbH, Market Segment Material Sciences, Munich Site, Germany
}

Graphene is a tightly packed layer of carbon atoms that are bonded together in a hexagonal honeycomb lattice. Only one atom thick (335 pm) but up to several micrometer in lateral extent, graphene shows many extraordinary material properties. As an electric conductor, it performs as good as copper. In the ability to transport heat, it outperforms all other known materials. Optically it is highly transparent but completely impermeable for any other atoms. Harder than steel and also showing a high elasticity, graphene unites several astonishing material properties. This makes graphene one of the most scientific investigated and promising materials for technical applications.

The graphene market will be split across many application sectors, like super capacitors, displays, wearable technology and printed electronics, each attracting a different type of graphene, thus various microscopic methods need to be used and combined to characterize the properties starting from the prepared graphene to the engineered graphene. Graphene is often prepared by using an adhesive tape. The top surface layers from Graphite can be removed and transferred to another surface. The most important question is, how thick the transferred flakes actually are and whether the process has left additional substances on the sample surface.

Two different procedures for preparing graphene are investigated. First, a basic approach with common adhesive tape and an uncleaned surface substrate and second a special adhesive wafer foil and plasma cleaned Si substrate is used. Two samples of graphene flakes were analyzed with light microscopy (LM), electron microscopy (EM) and atomic force microscopy (AFM) by means of the correlative microscopy technique (CorrMic). In a second step it is investigated, how optical interference contrast microscopy (TIC), cathodoluminescence (CL) and helium ion microscopy (HIM) can serve as complementary techniques in visualization, in profiling, and in measurement of conductivity of graphene.

The study demonstrates that it is possible to prepare few layer graphene flakes with both adhesive tape techniques. Bright field microscopy can indicate the number of graphene layers and thickness if the graphene is on a thin optically resonant film $[1,2,3]$. There is a strong difference in contamination between the two preparation methods. Standard adhesive tapes leave residuals on the surface and the flake itself, which often are only resolved by high resolution microscopic techniques like SEM and especially AFM. Since a lot of investigation on graphene is only done by using optical microscopy, such contamination may cause false and/or misleading results.

The findings also show that TIC-Profilometry allows for contact - free thickness measurement and profiling of graphene [4]. Correlating the height measured by AFM with the measured phase shift of a thin film is a method to measure refractive index, extinction, and conductivity. Even the energy dispersive back scattered detector (EsB) allows to distinguish between single and multi-layer graphene [5]. The detection of the finest monolayer thickness differences is possible from the BSE signal in the SEM as shown in this study. The noise is greatly reduced compared to the inelastic plasmon signal from the energy filtered signal in the TEM. The high surface sensitivity of the Helium Ion Microscope enables imaging of monolayers of graphene at high resolution. This is essential for the quality control of graphene 
patterning processes. By means of patterning and preparation of graphene nano ribbons one can create bandgaps in graphene which are necessary for electrical devices. The investigations showed that the bandgap in a graphene ribbon increases as the ribbon width decreases [6]. Graphene quantum dots on the other hand can be imaged with cathodoluminescence (CL). The CL spectrum has its peak were the quantum dots change their shape from circular-topolygonal-shape and their corresponding edge-state [7].

Graphene can be imaged with various microscopic methods, each revealing a different information. All methods have benefits and disadvantages, therefore using these methods in a combination often leads to deeper understanding of the graphene properties.

References:

[1] P. Blake et al , Appl. Phys. Lett. 91 (2007) 063124

[2] Z. H. Ni et al, Nano Lett. 7 (2007) 2758

[3] Y. Y. Wang et al, Nanotechnology 23 (2012) 495713

[4] M. Vaupel et al, J. Appl. Phys. 114 (2013) 183107

[5] Iwona Joswik-Biala et al, Microsc. Microanal. 20 Suppl 3 (2014)

[6] D.S. Pickard et al, Microscopy and Microanalysis 07/2012; 18(S2):800-801

[7] Soo Seok Kang et al, Current Applied Physics 14 (2014) S111-S114

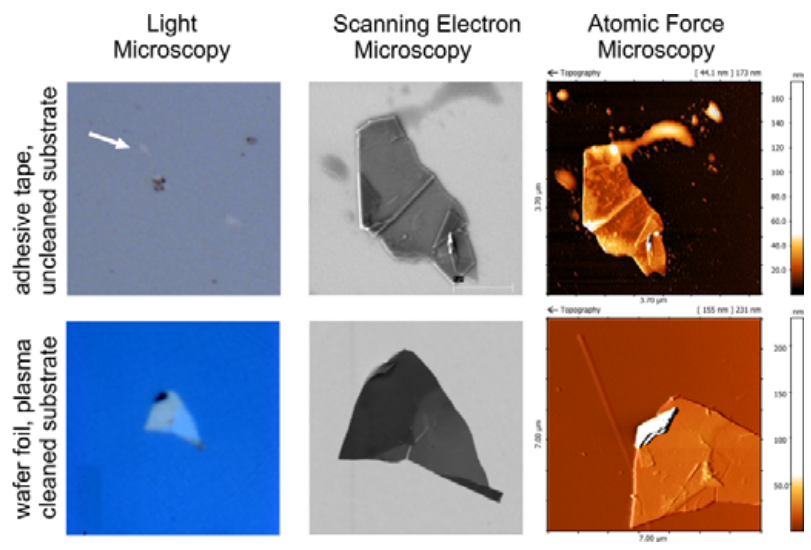

Figure 1. From LM to AFM; increasing resolution and decreasing speed of analysis
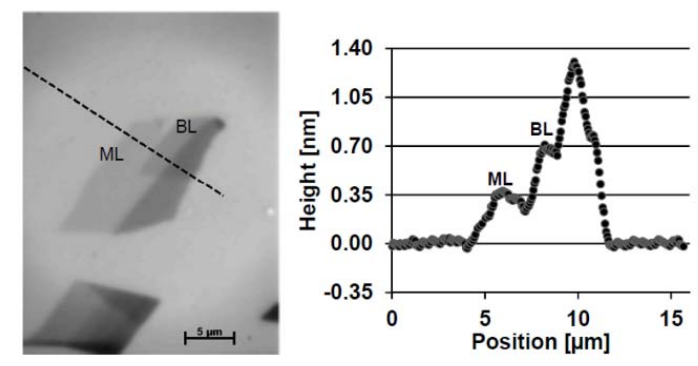

Figure 2. TIC microscopy: phase profile along dashed line, converted into height of graphene stack.

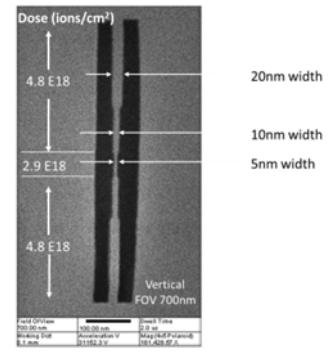

Figure 3. Nano ribbons in graphene. Created with the Helium Ion Microscope. Dr. Dan Pickard, National University of Singapore 